\title{
Keynote: Insight, Not (Random) Numbers: An Embedded Perspective
}

\author{
Thomas M. Conte \\ Center for Embedded Systems Resesrch \\ Department of Electrical and Computer Engineering \\ North Carolina State University
}

\begin{abstract}
Hamming said that the purpose of computing was "insight, not numbers." Yet benchmarking embedded systems is today a numbers game. In this talk, I will dissect Hamming's famous quote and provide some reasons to hope we can making benchmarking of embedded systems into a science. In particular, I will discuss how to model and measure quantities so that one can gain confidence in the results. I will use the industry standard EEMBC benchmark set as an example. Along the way, I will (I hope) give some insight into what the EEMBC benchmarks are trying to test.
\end{abstract}

